\title{
A novel small RNA CoaR regulates coenzyme $A$ biosynthesis and tolerance of Synechocystis sp. PCC6803 to 1-butanol possibly via promoter-directed transcriptional silencing
}

Tao Sun ${ }^{1,2,3}$, Guangsheng Pei ${ }^{1,2,3}$, Jiangxin Wang ${ }^{4}$, Lei Chen ${ }^{1,2,3^{*}}$ and Weiwen Zhang ${ }^{1,2,3,5^{*}}$

\begin{abstract}
Background: Microbial small RNAs (sRNAs) have been proposed as valuable regulatory elements for optimizing cellular metabolism for industrial purposes. However, little information is currently available on functional relevance of sRNAs to biofuels tolerance in cyanobacteria.

Results: Here, we described the identification and functional characterization of a novel 124 nt sRNA Ncl1460 involved in tolerance to biofuel 1-butanol in Synechocystis sp. PCC 6803. The expression of Ncl1460 was verified by blotting assay and its length was determined through 3' RACE. Further analysis showed that Ncl1460 was a negative regulator of s/r0847 (CoaD) and s/r0848 operon responsible for coenzyme A (CoA) synthesis possibly via promoterdirected transcriptional silencing mechanisms which has been widely discovered in eukaryote; thus Ncl1460 was designated as CoaR (CoA Biosynthesis Regulatory sRNA). The possible interaction between CoaR and target genes was suggested by CoA quantification and green fluorescent protein assays. Finally, a quantitative proteomics analysis showed that CoaR regulated tolerance to 1-butanol possibly by down-regulating CoA biosynthesis, resulting in a decrease of fatty acid metabolism and energy metabolism.
\end{abstract}

Conclusions: As the first reported sRNA involved CoA synthesis and 1-butanol tolerance in cyanobacteria, this study provides not only novel insights in regulating mechanisms of essential pathways in cyanobacteria, but also valuable target for biofuels tolerance and productivity modifications.

Keywords: sRNA, Synechocystis, Tolerance, 1-Butanol, CoA biosynthesis, Transcriptional silencing

\section{Background}

Bacterial small RNAs (sRNAs) are functional non-coding RNAs with a typical length of 50-300 nt, among which trans-acting sRNAs (ncRNAs) transcribe from the intergenic regions and function at a distance to alter the expression of target RNAs, while cis-acting sRNAs (asRNAs) transcribe from the complementary strand of the

\footnotetext{
*Correspondence: Ichen@tju.edu.cn; wwzhang8@tju.edu.cn ${ }^{1}$ Laboratory of Synthetic Microbiology, School of Chemical Engineering and Technology, Tianjin University, Tianjin 300072, People's Republic of China

Full list of author information is available at the end of the article
}

known open reading frames (ORFs) and regulate translation or decay mRNA molecules through base paring [1]. Photosynthetic cyanobacteria have been proposed as "autotrophic cell factories" for biofuels production due to its ability to utilize $\mathrm{CO}_{2}$ and sunlight directly for growth [2]. In cyanobacteria Synechocystis sp. PCC 6803 (hereafter Synechocystis), though previous studies have approximately identified 300 putative sRNAs [3], their functional characterizations still significantly lag behind. So far only several sRNAs of Synechocystis have been functionally revealed, including an asRNA IsrR functioning as a negative regulator of the $\mathrm{CP} 43$ homolog IsiA, a negative 
regulator named as_flv4 involved in response to shifts in inorganic carbon supply, PsbA2R and PsbA3R acting as positive regulators to enhance the stability of target mRNAs as well as PsrR1 controlling photosynthetic functions [4]. Recently, NsiR4 was found involved in nitrogen assimilation control [5].

1-Butanol biosynthetic pathways have been introduced into various cyanobacterial systems and its production could reach $404 \mathrm{mg} / \mathrm{L}$ in Synechococcus elongatus PCC 7942 and $37 \mathrm{mg} / \mathrm{L}$ in Synechocystis [6, 7]. However, current productivity is still much lower than the native Clostridium or even synthetic Escherichia coli systems $[8,9]$, partially due to the high toxicity of 1-butanol to cyanobacteria [2]. Recently, Kaczmarzyk et al. [10] overexpressed an RNA polymerase sigma factor sigB in Synechocystis and successfully enhanced the tolerance to 1-butanol in a butanol-shock experiment. Our recent efforts on studying metabolic responses of Synechocystis to various biofuels have also led to the discovery of two response regulator genes (i.e., slr1037 and sll0039) related to 1-butanol tolerance [11, 12], and several transcriptional regulators (i.e., sll0794, sll1392, sll1712, and slr1860) related to ethanol tolerance [13, 14]. These studies demonstrated the possibility of improving biofuel tolerance by engineering transcriptional regulation. Previous studies have shown the vital roles of sRNAs in responding to environmental perturbations among various microbes through transcriptional or post-transcriptional regulation [1]. In a recent study, Gaida et al. [15] showed that overexpression of three sRNAs, DsrA, RprA, and ArcZ, could increase acid tolerance of $E$. coli up to 8500-fold. Meanwhile, the transcriptomic analysis of cellular responses to ethanol production in Synechocystis identified several regulated functionally unknown sRNAs including ncl1740, ncl1390, and ncl1600 [16], suggesting that sRNAs could be involved in the regulation of biofuels tolerance.

In this work, we employed a RNA-seq approach to identify sRNAs related with exogenous 1-butanol tolerance in Synechocystis, which led to discover a novel sRNA Ncl1460 responsive to 1-butanol. Further analysis demonstrated that Ncl1460 was a negative regulator of coaD involving coenzyme A (CoA) biosynthetic pathway, and was accordingly designated as CoaR (CoA Biosynthesis Regulatory sRNA). The interaction between CoaR and target genes was verified by CoA quantification and GFP assays. Finally, quantitative proteomics analysis revealed the decreased CoA content caused by coaR overexpressing could down regulate fatty acid metabolism and energy metabolism thus led to a decreased 1-butanol tolerance. As the first reported sRNA involved CoA synthesis and 1-butanol tolerance in cyanobacteria, this study provides not only novel insights in regulating mechanisms of essential pathways in cyanobacteria, but also valuable target for biofuels tolerance and productivity modifications.

\section{Results \\ Growth of Synechocystis under 1-butanol stress}

Effects of 1-butanol on Synechocystis were performed using wild-type (WT) in BG-11 media supplemented without or with 1-butanol at a concentration of $0.15,0.20$ and $0.25 \%(v / v)$, respectively (Fig. 1a). Consistent with previous study [17], the results showed that $0.20 \%(v / v)$ 1-butanol caused about $50 \%$ growth inhibition at $48 \mathrm{~h}$, and this concentration was selected for the transcriptomic analysis. WT cultivated with or without 1-butanol $(0.20 \%, v / v)$ were collected at 24,48 , and 72 for small RNA-seq analysis, respectively, named accordingly as C24, C48, and C72 for control as well as B24, B48, and B72 for butanol-treated samples, respectively.f

\section{sRNome analysis}

After adapter trimming and data filtering, a total of 9,669,974, 13,383,938, 6,919,528, 9,938,132, 6,951,722, and $8,373,222$ reads were obtained in six samples (i.e., C24, C48, C72 and B24, B48, B72, respectively). Then the sRNA reference libraries of Synechocystis (including approximately 1,071 known asRNAs and 320 known ncRNAs) described previously by Mitschke et al. [3] were used to identify the sRNAs in our study. Although the effective sequencing depth may vary in different samples, the obtained reads were able to match to approximately 1000 asRNAs and 290 ncRNAs (Additional file 2: Table S1), suggesting a good coverage.

Reads per kilobase per million mapped reads (RPKM) was employed to normalize the raw reads and a principal component analysis (PCA) was used to visualize the sRNomic expression profiles. The score plot showed that 1-butanol-treated samples (i.e., B24, B48 and B72) were visibly separated from the control samples (i.e., C24, C48, and C72) (Fig. 1b), suggesting that significant responses existed after 1-butanol treatment. Comparative expression analysis of the identified sRNAs showed that a total of 98, 168, 121 asRNAs and 42, 60, 43 ncRNAs were differentially expressed at 24,48 , and $72 \mathrm{~h}$ after 1-butanol treatment, respectively, using a criterion of the fold change $>1.5$ and $p$ values $\leq 0.05$ (Additional file 2: Table S2). To validate the reliability, 10 sRNAs were randomly selected for a quantitative real-time RT-PCR (qRTPCR) analysis (Primers were listed in Additional file 2: Table S3.). Comparative qRT-PCR analysis between the butanol-treated and control samples showed a significant correlation with a correlation coefficient $R^{2}>0.70$ using the Pearson correlation analysis (Fig. 1c), suggesting the high reliability of the sRNomic analysis. 

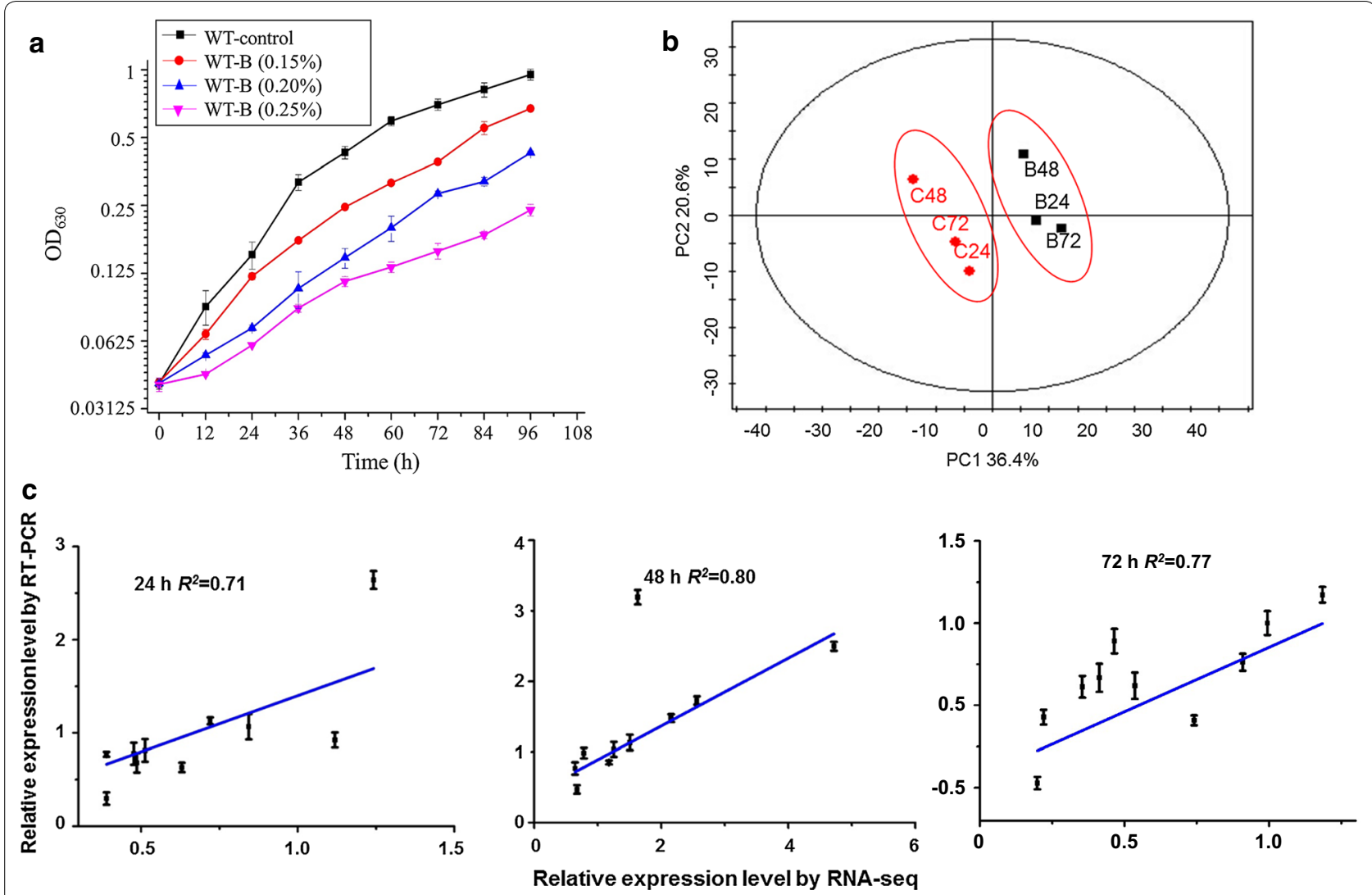

Fig. 1 a Growth curves of WT in BG11 media with or without different concentrations $(v / v)$ of 1-butanol. The error bars represent the calculated standard deviation of the measurements of three biological replicates. b PCA analysis of sRNome. C24, C48, C72 and B24, B48, B72 represent samples cultured at 24, 48, and $72 \mathrm{~h}$ in normal BG11 medium or BG11 medium supplemented with 1-butanol, respectively. c Validation of sRNome by qRT-PCR. The error bars represent the calculated standard deviation of the measurements of three biological replicates

sRNA Ncl1460 involved in 1-butanol stress response

A series of differentially regulated ncRNAs were selected for constructing overexpression [sense fragments of sRNA, expressed as sRNA(+)] and suppression strains [antisense fragments of sRNA, expressed as $\operatorname{sRNA}(-)$ ] using a replicating vector PJA2 [10] and the phenotypic difference was examined under $0.20 \%(v / v)$ 1-butanol stress (Additional file 2: Table S4; Additional file 1: Fig. S1A) [18]. Although most of the mutants constructed showed no growth difference from WT, Ncl1460(+) (i.e., Synechocystis with overexpressed ncl1460) became more sensitive to $0.20 \%(v / v)$ 1-butanol than WT (Fig. 2a). This phenotype was even more significant under high 1-butanol concentration like $0.25 \%(v / v)$ (Fig. 2b, c). Interestingly, Ncl1460(-) (i.e., Synechocystis with suppressed ncl1460) became more tolerant to 1-butanol than WT (Fig. 2a, b). The overexpression and suppression of $\mathrm{Ncl} 1460$ in $\mathrm{Ncl} 1460(+)$ and $\mathrm{Ncl} 1460(-)$ were confirmed by reverse transcriptional PCR (RT-PCR) using WT as a control (Additional file 1: Fig. S3A), which clearly showed that the transcriptional level was increased in Ncl1460(+) but decreased in Ncl1460(-), respectively.

The above phenotype indicated that $n c l 1460$ may be a negative regulator during 1-butanol stress response. In the sRNomic data, the RKPM values for Ncl1460 were $14.52,8.40$, and 11.37 under control condition at 24, 48, and $72 \mathrm{~h}$, respectively, while they respectively decreased to $5.65,5.66$, and 4.03 after 1 -butanol treatment, indicating a remarkably decrease of the relative expression by 2.5-, 1.5-, and 2.9-fold (Additional file 2: Table S1). To verify whether $\mathrm{Ncl1460(+)}$ was sensitive specifically to 1-butanol, growth patterns were also determined under $\mathrm{Cd}^{2+}(4.6 \mu \mathrm{M})$, low $\mathrm{pH}(\mathrm{pH}=6.1)$ and $\mathrm{NaCl}(4.0 \% \mathrm{w} / \mathrm{v})$ stress conditions. However, no differences were observed under $\mathrm{Cd}^{2+}, \mathrm{NaCl}$, and low $\mathrm{pH}$ conditions, thus we focused on 1-butanol tolerance in this study.

\section{Northern blotting and 3' RACE of Ncl1460}

To verify the expression of Ncl1460, northern blotting assays was conducted using the specific probe of Ncl1460 and the results shown in Additional file 1: Fig. S3B clearly 

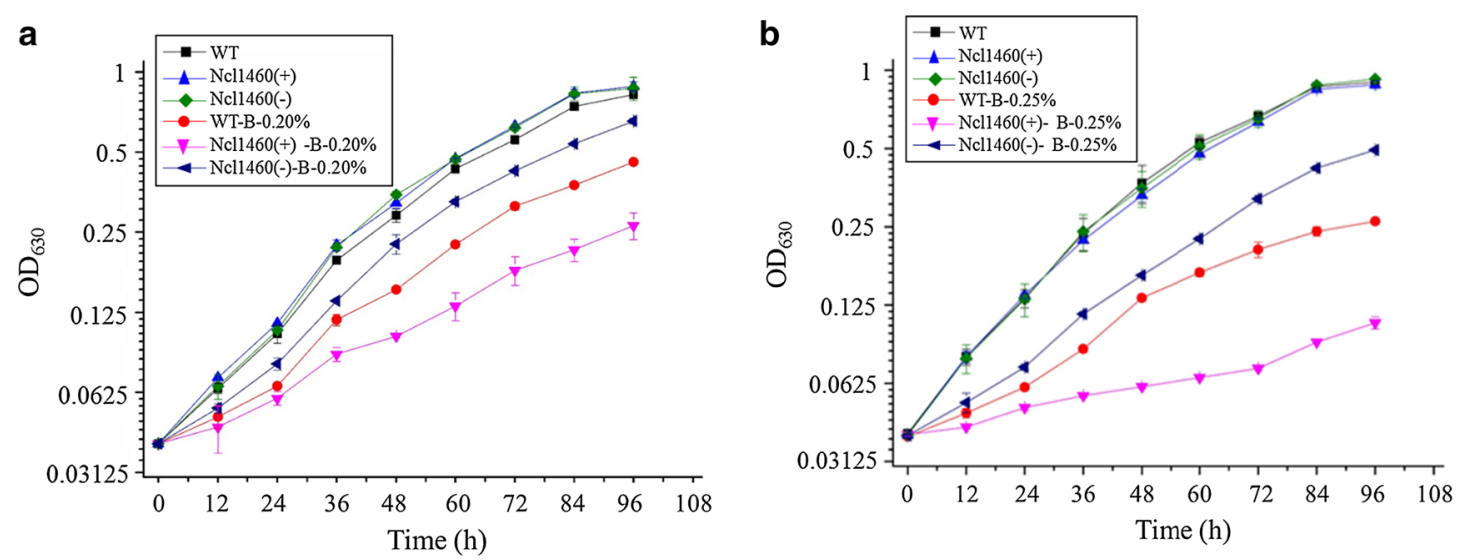

C

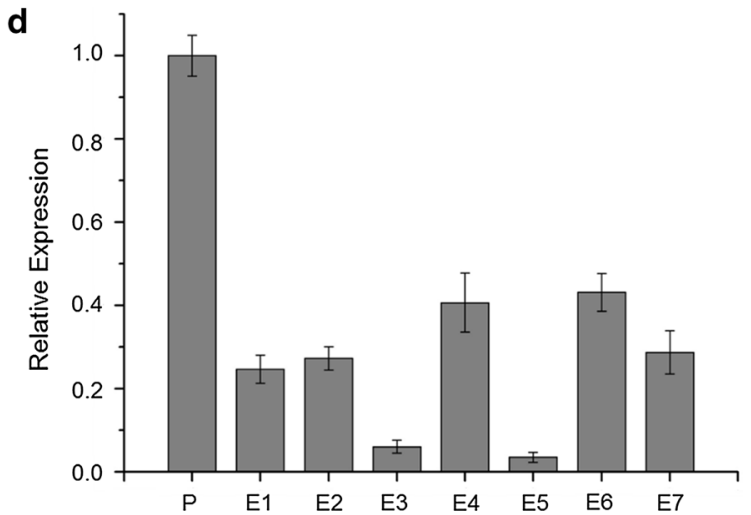

Fig. 2 a Growth patterns of WT, Ncl1460(+), and Ncl1460(-) in BG1 1 media with or without $0.20 \%(v / V)$ 1-butanol. The error bars represent the calculated standard deviation of the measurements of three biological replicates. b Growth patterns of WT, Ncl1460(+), and Ncl1460(-) in normal BG11 media with or BG11 without $0.25 \%(v / V)$ 1-butanol. The error bars represent the calculated standard deviation of the measurements of three biological replicates. c Growth phenotypes of WT, Ncl1460(+), and Ncl1460(-) in BG11 medium with 0.25\% (v/V) 1-butanol. d Relative expression levels of Ncl1460 in selected butanol-adapted strains to WT. E1, E2, E3, E4, E5, E6, and E7, respectively, represent the butanol-adapted strains that endure $0.20,0.25,0.30,0.35,0.40,0.45$, and $0.50 \%$ 1-butanol $(\mathrm{v} / \mathrm{V})$. The error bars represent the calculated standard deviation of the measurements of three biological replicates

demonstrated its presence as a sRNA. In a previous study, the $5^{\prime}$ end of Ncl1460 has been determined while its $3^{\prime}$ end reminded unclear since the longest transcript among the several candidate transcripts for ncl1460 through deep sequencing was simply selected in the previous study [19]; here, we determined the $3^{\prime}$ end using $3^{\prime}$ RACE (Additional file 1: Fig. S3C). After Sanger sequencing, the corrected length of Ncl1460 was confirmed as $124 \mathrm{nt}$, which was $55 \mathrm{nt}$ shorter than previously reported (179 nt) [3].

\section{sRNA Ncl1460 up-regulated in butanol-adapted strains}

In our previous study, Wang et al. [20] achieved a $150 \%$ increase in tolerance to 1-butanol in Synechocystis after a continuous 94 passages with gradually increased 1-butanol concentration from 0.20 to $0.50 \%(v / v)$. As ncl1460 involve in 1-butanol tolerance, its expression may also be regulated during the evolutionary process. To prove the hypothesis, we applied a qRT-PCR analysis to measure the relative expression levels of $n c l 1460$ in seven evolutionary strains enduring different concentrations of 1-butanol. The results showed that relative expression level of ncl1460 decreased clearly in all the evolutionary strains, and it was even lower in E3 and E5 which can endure 0.30 and $0.40 \%$ 1-butanol $(v / v)$ than that in WT, respectively, (Fig. 2d), suggesting that besides coding genes, the transcriptional level of non-coding sRNAs could also change in laboratory-based adaptive strains.

\section{sRNA Ncl1460/CoaR as a negative regulator for coenzyme A biosynthesis}

ncl1460 was located between genes encoding a phytochrome-like protein (Sll0821) and a phosphopantetheine adenylyltransferase (PPAT, Slr0847) (Fig. 3a). To identify potential target genes regulated by Ncl1460, the CopraRNA software developed by Wright et al. (2014) was employed for target prediction [21] and six 

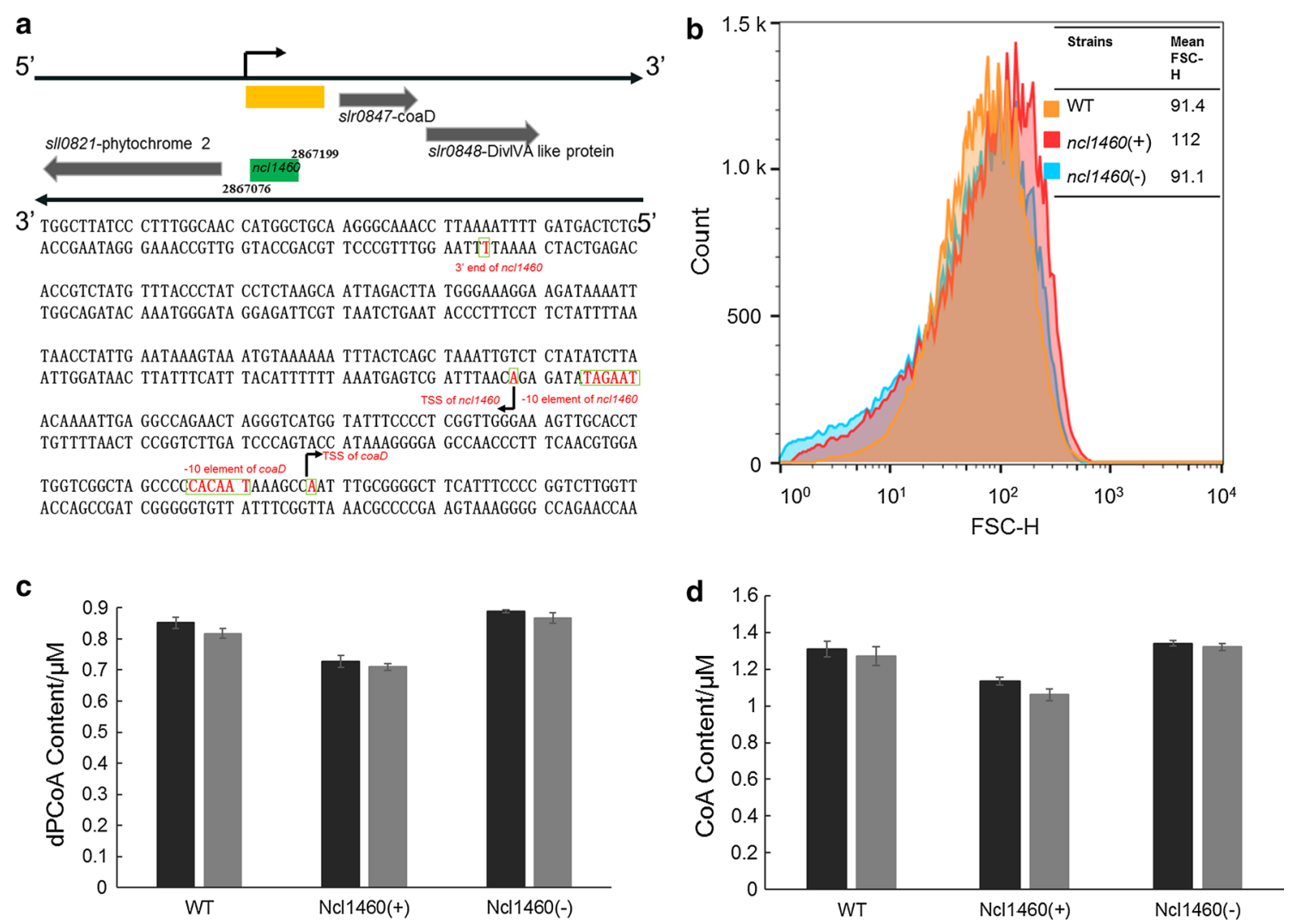

Fig. 3 a Genetic location and detailed sequence information of Ncl1460 in the Synechocystis genome. b Cell size measurements of the WT, $\mathrm{Ncl} 1460(+)$ and Ncl1460(-) strains. c Quantitation of dPCoA in the WT, Ncl1460(+) and Ncl1460(-) strains. d Quantitation of CoA in the WT, $\mathrm{Ncl} 1460(+)$ and $\mathrm{Ncl1460(-)} \mathrm{strains.} \mathrm{Samples} \mathrm{were} \mathrm{harvested} \mathrm{at} 48 \mathrm{~h}$ and the error bars represent the calculated standard deviation of the measurements of three biological replicates

cyanobacteria genomes (i.e., NC_000911, NC_008312, NC_009091, NC_009840, NC_011738, NC_007776) were taken as the sRNA homologs for inputs [22]. The result found one of the flanking genes slr0847 ranked first in the candidates (Additional file 1: Fig. S4). In Synechocystis, PPAT encoded by slr0847 was the only enzyme reported so far to catalyze the conversion from pantotheine $4^{\prime}$-phosphate to $3^{\prime}$-dephospho-CoA (dPCoA) in CoA biosynthesis [23]. In addition, slr0847 was found to be organized into one possible operon with slr0848 encoding a hypothetical protein which led to an enlarged cell size after deletion $[24,25]$. As ncl1460 located upstream the slr0847 on the complementary strand, we hypothesized that Ncl1460 may regulate $s l r 0847$ by directly base paring. Transcriptional starting site (TSS) of $s l r 0847$ has been identified in previous study and Ncl1460 was on its upstream but not within the transcribed region of slr0847 (Fig. 3a), excluding the possibility that Ncl1460 regulated slr0847 through post-transcriptional mechanisms by base paring [19]. As the possible interaction region between $n c l 1460$ and upstream region of slr0847 was clearly predicted by CopraRNA (Additional file 1: Fig. S5), suggesting other mechanism may exist. In eukaryotes like Saccharomyces cerevisiae, plants and even mammalian cells, promoter-directed transcriptional silencing mediated by siRNA, have been discovered in recent years [26]. Under this mechanism, sRNA located beyond the TSS of target genes and silenced targets through mediating the methylation of promoters [27]. To verify whether Ncl1460 could affect its potential targets slr0847 and slr0848, we examined the intracellular contents of $\mathrm{dPCoA}, \mathrm{CoA}$ as well as the cell size differences between WT, Ncl1460(+) and Ncl1460(-) under both normal BG11 or BG11 medium with $0.25 \%(v / v)$ 1-butanol. Using liquid chromatography-mass spectrometry (LC/MS), the intracellular contents of dPCoA and $\mathrm{CoA}$ in $\mathrm{Ncl} 1460(+)$ strain were found decreased by 14.7 and $13.5 \%$ under normal medium as well as 13.1 and $15.5 \%$ under 1-butanol stress condition, respectively. Although it is not significant, their abundances especially the abundance of $\mathrm{dPCoA}$ were increased by 4 and $6 \%$ in Ncl1460(-) strain compared with WT respectively under 
normal medium or 1-butanol stress condition (Fig. 3c, d). Furthermore, flow cytometry analysis showed that the mean size of Ncl1460(+) was enlarged than that of WT while the size of $\mathrm{Ncl} 1460(-)$ cells showed no significant difference (Fig. 3b), consistent with the previous report [25]. These results further indicated that Ncl1460 was a negative regulator of slr0847 and slr0848 operon which could affect the CoA biosynthesis and cell size; thus, the sRNA Ncl1460 was renamed as CoaR (CoA Biosynthesis Regulatory sRNA).

\section{GFP assays in Synechocystis}

To demonstrate the possible interaction between CoaR and the promoter of slr0847, the whole intergenic sequence between sll0821 and slr0847 (containing the promoter of $s l r 0847$ named $\mathrm{P}_{\text {slro847 }}$ ) was amplified and fused to the GFP coding gene. The fused fragment was then ligated to backbone of pXT37b (without original promoter, Additional file 1: Fig. S1B) and introduced into WT, CoaR(+) and CoaR(-) strains (Fig. 4a). Full segregation of the transformants (i.e., WT-GFP, CoaR(+)-GFP, and CoaR(-)-GFP) were achieved by consecutively passaging and verified by PCR (Fig. 4b). Fluorescence of WT-GFP, CoaR(+)-GFP, and CoaR(-)-GFP was measured after cultivation for $48 \mathrm{~h}$ using a HITACHI F-2700 fluorescence spectrophotometer. The fluorescence intensity was normalized by optical density $\left(\mathrm{OD}_{730}\right)$ of each sample and the basal fluorescence of WT. The results illustrated in Fig. 4c showed that the fluorescence intensity of CoaR(+)-GFP was decreased significantly than that of WT-GFP and CoaR(-)-GFP, demonstrating that the function of CoaR may functioned by silencing the promoter region of $s l r 0847$.

\section{Quantitative proteomics and lipid profiles reveals possible tolerance mechanisms}

Finally, a quantitative proteomic analysis was utilized to identify the differential metabolic responses to 1-butanol between the WT and CoaR $(+)$ strains. Samples of WT and $\operatorname{CoaR}(+)$ were harvested after cultivation with $(0.20 \%, v / v) 1$-butanol for $48 \mathrm{~h}$, each with two biological replicates for proteomic analysis. After data filtering, the qualified spectra were matched to 2036 proteins (Additional file 1: Fig. S7). Comparative analysis showed that 159 proteins were down-regulated while 166 proteins up-regulated in the $\operatorname{CoaR}(+)$ strain using a 1.2fold change as cut-off ( $p$ value $<0.05$ ) compared to WT (Additional file 2: Table S5). Proteomic data showed that the relative abundance of Slr0847 was decreased by $20 \%$ in the CoaR $(+)$ strain, in good agreement with the $15.5 \%$ decrease of the CoA content in the same cells. $\mathrm{CoA}$ is an essential cofactor for numerous metabolic and

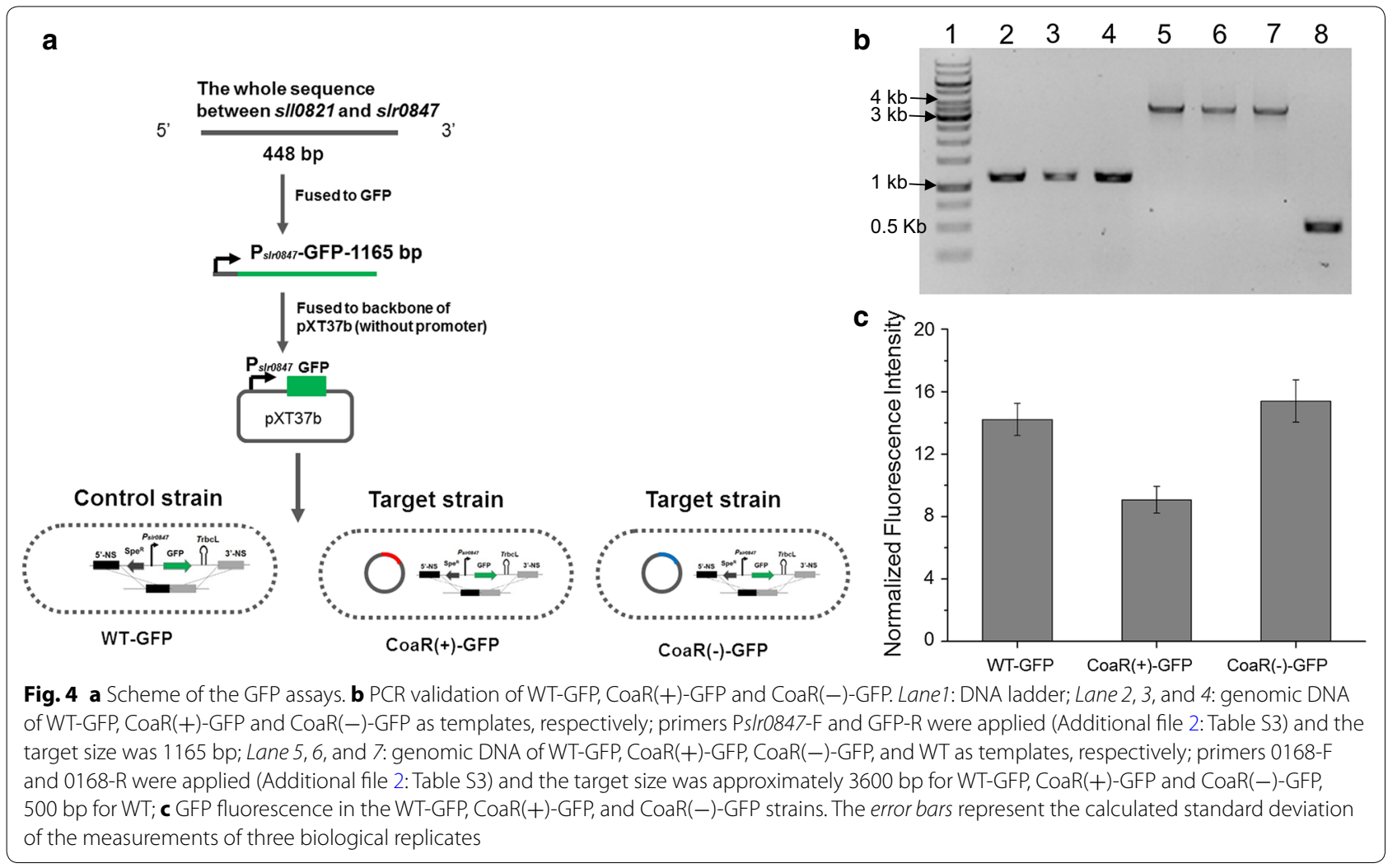


energy-yielding reactions, also notable for its role in fatty acids metabolism and energy metabolism [28]. Consistently, the proteomic results showed that a wide range of metabolic changes including fatty acids metabolism, energy metabolism, amino acids metabolism, signal conduction, $\mathrm{ABC}$ transporters, ribosome metabolism, and cofactor biosynthesis were differentially regulated in the $\mathrm{CoaR}(+)$ strain, probably directly or indirectly due to the decreased cellular content of CoA in $\mathrm{CoaR}(+)$ under 1-butanol stress (Fig. 5).

The changes of intercellular CoA abundance could directly affect fatty acids biosynthesis and amino acids metabolism, and these metabolisms have been found related to tolerance to biofuels previously [29]. MalonylCoA-acyl carrier protein transacylase (FabD, Slr2023) was found down-regulated in CoaR(+) (Fig. 5). FabD was a key enzyme in the fatty-acid biosynthesis pathway, catalyzing the transfer of a malonyl moiety from malonyl-CoA to holo acyl carrier protein (ACP) [30]. Early studies have shown that increased long-chain fatty acids could enhance biofuel tolerance like ethanol in Thermoanaerobacter ethanolicus [31]. In addition, we also found that serine hydroxymethyltransferase (GylA) and phosphoglycerate mutase (Slr1748) involving glycine, serine, and threonine metabolism were down-regulated in the CoaR(+) strain and they have been found up-regulated in Synechocystis upon 1-butanol stress previously [17]. To determine whether the fatty acid biosynthesis was affected, we measured the total lipid profiles of WT, CoaR(+), and CoaR(-). Total lipids were extracted from approximately $5 \mathrm{mg}$ dry cells of WT, CoaR(+), and CoaR(-), each with 3 biological replicates, then subjected to gas chromatography (GC) analysis following the protocol of Liu et al. [32]. The peak areas of each fatty acid were normalized by the dry weight of each sample. The results showed that the total lipid per milligram dry weight in the $\mathrm{CoaR}(+)$ strain was decreased by approximately $7.0 \%$, compared with WT (Table 1 ). In addition, components of C14:1n9, C16:0, C18:1n9, C18:2n6, and C18:3n6 were found with a slight decrease in the $\mathrm{CoaR}(+)$ strain compared to WT. Interestingly, although the total lipid content in the CoaR(-) strain was approximately the same as that in WT, C14:1n9 and $\mathrm{C} 18: 3 \mathrm{n} 6$ decreased in $\mathrm{CoaR}(+)$ were increased in CoaR(-), which could be resulted from the repression of coaR in CoaR $(-)$, consistent with the previous study in Haematococcus pluvialis finding the relative abundance of C18:3n6 was increased in cells under nitrogen depletion and low-temperature conditions [33].

The energy metabolism is also directly related with CoA [28]. Four proteins (i.e., Slr0343, Sll1796, Ssl3093, and Sll1484) related with energy metabolism and photosynthesis process were down-regulated in the butanol sensitive CoaR(+) strain. Slr0343 (PetD) and Sll1796

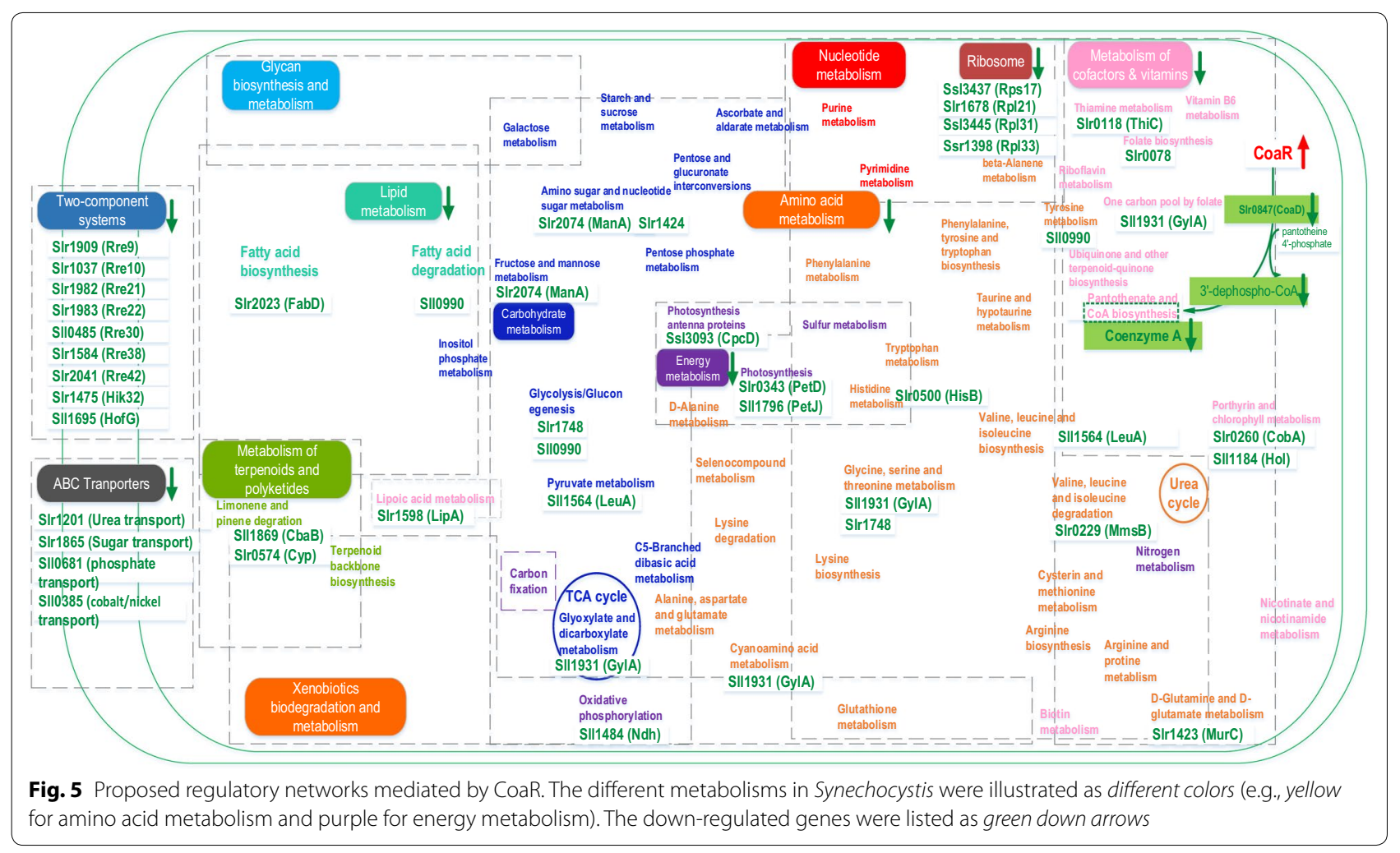


Table 1 Lipid profiles of WT, CoaR(+), and $\operatorname{CoaR}(-)$

\begin{tabular}{lccccc}
\hline & WT & CoaR(+) & CoaR(-) & CoaR(+)/WT & CoaR(-)/WT \\
\hline C14:1n9 & $0.85 \pm 0.027$ & $0.82 \pm 0.056$ & $0.87 \pm 0.038$ & $0.96 \pm 0.073$ & $1.02 \pm 0.055$ \\
C16:0 & $26.77 \pm 0.087$ & $23.99 \pm 0.99$ & $25.92 \pm 0.13$ & $0.9 \pm 0.037$ & $0.97 \pm 0.006$ \\
C16:1n7 & $3.69 \pm 0.031$ & $3.67 \pm 0.20$ & $3.68 \pm 0.24$ & $0.99 \pm 0.055$ & $1 \pm 0.066$ \\
C16:2n4 & $0.35 \pm 0.004$ & $0.37 \pm 0.025$ & $0.43 \pm 0.042$ & $1.05 \pm 0.073$ & $1.23 \pm 0.12$ \\
C16:4n4 & $0.53 \pm 0.024$ & $0.55 \pm 0.017$ & $0.52 \pm 0.033$ & $1.03 \pm 0.056$ & $0.97 \pm 0.076$ \\
C18:1n9 & $1.37 \pm 0.058$ & $1.32 \pm 0.08$ & $1.46 \pm 0.056$ & $0.96 \pm 0.071$ & $1.06 \pm 0.061$ \\
C18:1n7 & $0.28 \pm 0.029$ & $0.37 \pm 0.018$ & $0.45 \pm 0.003$ & $1.33 \pm 0.15$ & $1.62 \pm 0.17$ \\
C18:2n6 & $7.88 \pm 0.69$ & $7.39 \pm 0.48$ & $7.75 \pm 0.52$ & $0.94 \pm 0.10$ & $0.98 \pm 0.11$ \\
C18:3n6 & $7.26 \pm 0.21$ & $7.00 \pm 0.52$ & $7.56 \pm 0.44$ & $0.96 \pm 0.077$ & $1.04 \pm 0.068$ \\
Total area & $48.97 \pm 1.23$ & $45.47 \pm 1.26$ & $48.61 \pm 1.16$ & $0.93 \pm 0.035$ & $0.99 \pm 0.034$ \\
\hline
\end{tabular}

Content of each type of fatty acids was shown as the normalized peak area (area/mg) by GC. The first three columns were the mean peak area and the standard deviation of each fatty acid as well as the total area from three replicates. The last two columns represent the relative content of each fatty acid as well as the total lipid in $\mathrm{CoaR}(+)$ or $\mathrm{CoaR}(-)$ to that in WT

(Pet) were important parts of cytochromes and played vital roles in electron transfer [34]. Ssl3093 (CpcD) is a phycobilisome small rod linker polypeptide and involved in light harvesting. Sll1484 (Ndh) is a type-2 NADH dehydrogenase serving as redox-active electron transport intermediates [35]. Up-regulation of Slr0343 was reported previously under thermo-stress condition and its increased abundance allowed for rapid electron transfer from PSII to PSI [35]. In addition, Sll1796 was found significantly up-regulated in our previous transcriptomic and proteomic studies of Synechocystis under 1-butaonl stress $[17,36]$, and Ssl3093 was found significantly upregulated in Synechocystis upon ethanol stress [37].

Besides the pathways directly related with CoA, five $\mathrm{ABC}$ transporters and nine two-component signal transduction system (TCS) proteins were found down-regulated in the butanol sensitive CoaR(+) strain (Fig. 5). $A B C$ transport proteins have been suggested as an important mechanism against biofuel toxicity [38]. Twocomponent systems have been proved involving regulation of multiple stress conditions [39]. Chen et al. [11] recently found that the deletion of slr1037 gene encoding a response regulator in Synechocystis led to a decreased tolerance to 1-butanol; consistently, our proteomic analysis also showed that Slr1037 was down-regulated in $\mathrm{CoaR}(+)$. Nevertheless, it is still unclear of the functional relationship between CoaR and Slr1037.

\section{Discussion}

It is estimated that CoA is utilized by $9 \%$ of known enzymes and involved in over 100 reactions in cells [40]. In bacteria, CoA biosynthesis includes five enzymatic steps starting from pantothenate (Fig. 6) [41]. Given the fact that $\mathrm{CoA}$ is an essential cofactor in all living organisms [42], its regulation has attracted significant attentions. Early studies found that pantothenate kinase (CoaA) catalyzing the first step as well as phosphopantetheine adenylyltransferase (CoaD) in the fourth step were two key regulatory points in CoA biosynthesis process [43] (Fig. 6). Homologues of the coaD gene have been identified in a wide range of bacterial species, sharing a similar size (140-169 residues) and a high degree of sequence homology (32-52\% identical) to the E. coli counterpart [44]. In addition, an early study showed that expression of coaD was regulated through a feedback of end-product CoA in Pseudomonas aeruginosa [45]. For Synechocystis, although no experimental information is available, slr0847 is annotated as coaD involved in catalyzing the penultimate step of the CoA synthesis [23]. Besides, the identity between Slr0847 and E. coli $\mathrm{CoaD}$ was $48 \%$ as revealed by Blast-p (http://blast.ncbi. nlm.nih.gov/Blast.cgi?PAGE=Proteins). The function of slr0847 homologue has been investigated in Arabidopsis thaliana through suppression, complementation, and overexpression [41], and the results showed that with a 90\% reduction of CoaD in $A$. thaliana, root growth, seed production and the fatty acid content were all negatively affected while the complementation of this gene recovered these effects, suggesting the essential roles of coaD in growth and fatty acid biosynthesis in plants [41]. So far no sRNA has been reported for regulatory roles in CoA biosynthesis in any species to our knowledge. Thus, CoaR could be the first sRNA with direct roles in regulating CoA biosynthesis in model cyanobacterium Synechocystis.

Significant increase of tolerance against salt and osmotic stresses has previously been observed when overexpressing coaD in $A$. thaliana, suggesting coaD and CoA biosynthesis could be related with responding to environmental stress [41]. Consistently, the decreased 


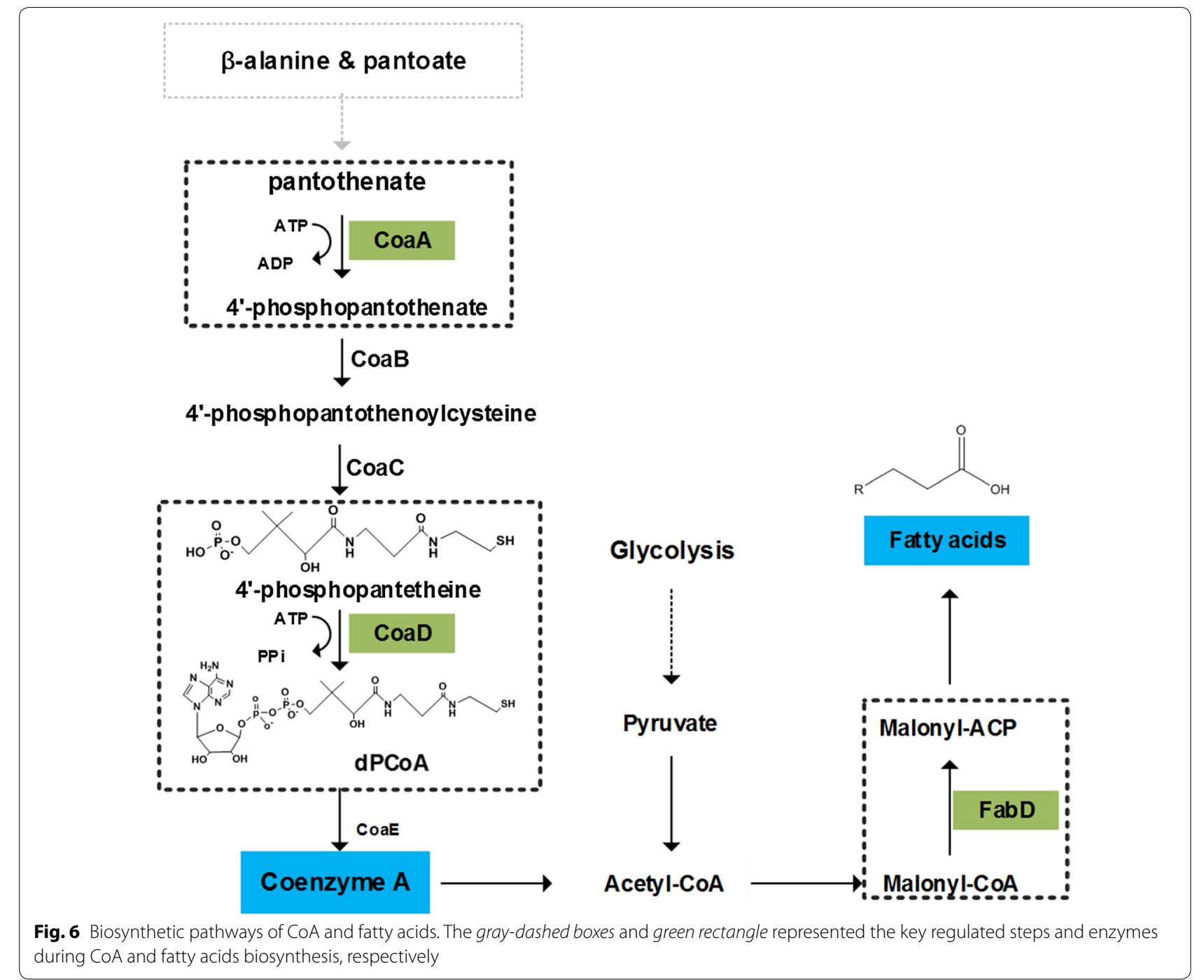

expression of coaD by overexpressing coaR led to a decreased tolerance to 1-butanol while suppressing coaR resulted in higher tolerance to 1-butanol. Although the $\mathrm{dPCoA}$ and $\mathrm{CoA}$ changes were not significant (which may be due to the low abundances of intracellular $\mathrm{dPCoA}$ and $\mathrm{CoA})$, the $\mathrm{CPCoA}$ and $\mathrm{CoA}$ contents in CoaR(-) had a slight increase, when compared with WT (Fig. 3c, d). Roles of sRNAs in biofuel stresses have been reported in other species. Venkataramanan et al. [46] recently discovered 159 sRNAs in responding to the native but toxic metabolites 1-butanol and butyrate in $C$. acetobutylicum. In addition, Cho et al. [47] validated 3 sRNAs (i.e., Zms2, Zms6, and Zms18) with differential expression in $Z$. mobilis under $5 \%$ ethanol stress. In addition, Aguilar et al. [48] found that sRNAs omrA and omrB regulating protein composition of the outer membrane were sitemutated after an adaptive laboratory evolution process of $E$. coli in glucose for $120 \mathrm{~h}$. Here we found the transcriptional level of coaR also changed in 1-butanol adaptive evolution process, suggesting the important roles of sRNAs in biofuels response. Unlike overexpression of genes, regulation mediated by sRNA hardly imposes any metabolic burden on cells, and represents a more effective strategy for strains modification [15]. Here we identified the sRNA CoaR related to biofuel tolerance through regulating $\mathrm{CoA}$ biosynthesis pathways, constituting a valuable basis for further sRNA engineering in cyanobacteria in the future.

Our studies via quantitative proteomics showed that CoA was involved in the 1-butanol stress response mainly through affecting the fatty acid biosynthesis and energy metabolism. In Bacillus subtilis, fatty acid biosynthetic genes including the $f a b$ initiation genes $f a b H B$ and $f a b D$ as well as the elongation genes $f a b F, f a b G$, and 
fabI were found significantly up-regulated after sorbic acid exposure [49]. Consistently, our proteomics analysis also revealed the reduction of FadD in the CoaR $(+)$ strain after 1-butanol exposure, and the down-regulation of FadD caused by decreased CoA may affect the membrane remodeling thus contributed to the low 1-butanol tolerance. In a study of $E$. coli in responding to heat shock stress, it was shown that intracellular ATP levels could transiently increase to meet the higher consumption for protein- and DNA-repair mechanisms [50]; besides, decreased expression of amino acyl-tRNA synthetases might promote the release of amino acids that feed energy-providing pathways [51]. Consistently, we found in this study, the decreased abundance of energy metabolism related proteins (i.e., Slr0343, Sll1796, Ssl3093 and Sll1484) and ribosome metabolism related proteins (i.e., Rps17, Rpl21, Rpl31 and Rpl33), could also lead to a sensitive phenotype of $\mathrm{CoaR}(+)$ to 1-butanol.

Finally, although we proposed a possible mechanism for CoaR based on our study, we cannot exclude other possible mechanisms as the direct interaction mechanism between $n c l 1460$ and slr0847 has not been verified experimentally. Nevertheless, this mode of mechanism has been well studied and demonstrated in eukaryotes like yeast, plants and mammalian cells [26]. In addition, previous studies found that almost all relevant regulatory genes can only affect tolerance at a small degree individually in Synechocystis suggesting that the tolerance may be regulated by multiple regulatory genes simultaneously [11-14], which is also consistent with the excellent results of enhancing acid tolerance significantly by overexpressing three sRNAs (DsrA, RprA, and ArcZ) in E. coli [15]. We believe with more of the tolerance-relevant sRNAs identified in the future, together with this finding, a better understanding of the tolerance regulation in Synechocystis will be eventually achieved.

\section{Conclusions}

This study found a novel sRNA CoaR regulating CoA synthesis as well as the tolerance of Synechocystis to 1-butanol. Overall, the study provided novel insight to the regulation of CoA biosynthesis in cyanobacteria, and also indicated that sRNAs could be useful targets in improving biofuel tolerance.

\section{Methods}

\section{Bacterial growth conditions}

For Synechocystis, WT and mutants were grown on BG11 agar plate or in medium $(\mathrm{pH}$ 7.5) under a light intensity of approximately $50 \mu \mathrm{mol}$ photons $\mathrm{m}^{-2} \mathrm{~s}^{-1}$ in an illuminating incubator or shaking incubator at $130 \mathrm{rpm}$ at $30{ }^{\circ} \mathrm{C}$ [17]. Medium for different mutants in this study was supplemented with appropriate antibiotic(s) to maintain plasmids with sRNAs or genes fused to genome (i.e., $10 \mu \mathrm{g} / \mathrm{mL}$ kanamycin, $10 \mu \mathrm{g} /$ $\mathrm{mL}$ spectinomycin or a combination). For E. coli, DH $5 \alpha$ were grown on LB agar plate or in LB medium with appropriate antibiotic(s) to maintain plasmids (i.e., $100 \mu \mathrm{g} / \mathrm{mL}$ ampicillin) at $37^{\circ} \mathrm{C}$ using incubator or shaking incubator at $200 \mathrm{rpm}$, respectively.

\section{SRNome sequencing and data analysis}

Small RNA-seq transcriptome sequencing was carried out as described previously [17]: (1) Samples composition: WT grew under BG11 medium with or without $0.20 \%(v / v)$ 1-butanol were collected for the transcriptome analysis at 24,48 , and $72 \mathrm{~h}$, respectively; (2) RNA preparation and cDNA synthesis: approximately $10 \mathrm{mg}$ of cell pellets was frozen by liquid nitrogen immediately after centrifugation. Total RNA extraction of 6 samples was achieved through a miRNeasy Mini Kit (Qiagen, Valencia, CA) following the protocols, and $500 \mathrm{ng}$ total RNA was subjected to cDNA synthesis using a NuGEN Ovation ${ }^{\circledR}$ Prokaryotic RNA-seq System (NuGEN, San Carlos, CA); (3) RNA sequencing: RNA $2 \times 100$ bp paired-end sequencing was performed using Illumina's Solexa Genome Analyzer II using the standard protocol. The image deconvolution and calculation of quality value were performed using Goat module (Firecrest v.1.4.0 and Bustard v.1.4.0 programs) of Illumina pipeline v.1.4. Sequenced reads were generated by base calling using the Illumina standard pipeline.

Genome sequence and sRNA annotation information of Synechocystis were downloaded from NCBI and Mitschke et al. [3]. sRNA sequence reads were pre-processed using NGS QC Toolkit (Version: 2.3) to remove low-quality bases and adapter sequences. Reads after QC were aligned to the Synechocystis genome using BurrowsWheeler Alignment tool software version 0.7.10 with perfect match parameters. Raw counts of reads that uniquely mapped to each sRNA region were calculated by HTseq (Version: 0.6.1). Then read counts were normalized to the aligned RPKM to obtain the relative expression levels.

\section{qRT-PCR analysis}

The RNA extraction method was the same as described above. cDNAs were synthesized using SuperScript ${ }^{\circledR}$ VILO $^{\mathrm{TM}}$ cDNA Synthesis Kit following manufacturer's protocol (Invitrogen, Carlsbad, CA) and $1 \mu \mathrm{L}$ of each dilution was used as template for following qRT-PCR reaction. The qPCR reaction was carried out in $10 \mu \mathrm{L}$ reactions containing $5 \mu \mathrm{L}$ of UltraSYBR Mixture $(\mathrm{CW}$

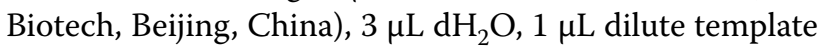
cDNA, and $1 \mu \mathrm{L}$ of each PCR primer, employing the StepOnePlus ${ }^{\mathrm{TM}}$ Real-Time PCR System (Applied Biosystems, Foster City, CA) [36]. Three technical replicates were 
performed for each sample. Data analysis was carried out using the StepOnePlus analytical software (Applied Biosystems, Foster City, CA) and the $2^{-\Delta \Delta C T}$ method [52]. $16 \mathrm{~S}$ rRNA was selected as a reference gene, and the data were presented as ratios of the amount of normalized transcript in the treatment to that from the WT control.

\section{RT-PCR analysis}

The RNA extraction method was the same as described above. cDNAs were synthesized using specific reverse primer of Ncl1460 named qRT-ncl1460-R (Additional file 2: Table S3) and SuperScript ${ }^{\circledR}$ VILO $^{\text {TM }}$ cDNA Synthesis Kit following manufacturer's protocol (Invitrogen, Carlsbad, CA). Then $1 \mu \mathrm{L}$ cDNA was used as template for RT-PCR using primer qRT-ncl1460-F and qRT-ncl1460-R.

\section{Northern blotting}

The non-radioactive northern blot method to detect small RNAs was performed following Kim et al. (2010)'s work [53]. Briefly, $10 \mu \mathrm{g}$ total RNA was extracted from WT for blotting assays. Oligonucleotides probe of CoaR (5'-GTTAAGATATAGAGACAAT T TAGCTGA-3') was added digoxin-label using Roche DIG Oligonucleotide Tailing Kit II (F. Hoffmann-La Roche Ltd, Switzerland) for hybridization. Anti-digoxigenin-AP and CSPD (F. Hoffmann-La Roche Ltd, Switzerland) were used to detection.

\section{3' Race}

Total RNA of WT was extracted as described above. Then RNA was added with a poly(A) tail using NEB $E$. coli poly(A) polymerase (New England Biolabs Inc., MA, USA). After that a specific primer containing oligo dT (5'-CACACAGGAAACAGCTATGACCATGTTTTTTTTTTTTTTTTTT-3') and SuperScript ${ }^{\circledR}$ VILO $^{\mathrm{TM}}$ cDNA Synthesis Kit were used to synthesize cDNA. At last, forward primer (5'-CCTTTCCCATAAGTCTAATTGCTTAGAGG-3') and reverse primer (5'-CACACAGGAAACAGCTATGACCATG-3') were used to amplify Ncl1460 containing $3^{\prime}$ end. PCR product was purified and ligated into pTZ57R/T using InsTAclone PCR Cloning Kit (Thermo Fisher scientific Inc., MA, USA) for sequencing analysis.

\section{qRT-PCR for adaptive laboratory evolutionary strains}

In this study, seven evolved strains from previous studies [20], i.e., E1, E2, E3, E4, E5, E6, and E7 which could, respectively, endure $0.20,0.25,0.30,0.35,0.40,0.45$, and $0.50 \%(v / v) 1$-butanol, were selected and cultured in normal BG11 medium along with WT and all the samples were harvested at $48 \mathrm{~h}$ for sRNA extraction and qRTPCR analysis.

\section{Mutant strains construction}

Escherichia coli DH5 $\alpha$ strain was used for vectors construction and enrichment. Primers used in this study were listed in Additional file 2: Table S2. All the plasmids and mutants constructed in this study were concluded in Additional file 2: Table S6. For overexpression and suppression of sRNAs, fragments of sRNAs and related reverse complementary chain were amplified from the Synechocystis genome with specific primers that introduced $X b a \mathrm{I}$ and $B a m H \mathrm{I}$ restriction sites and ligated to a broad host replicating vector pJA2 with anti-kanamycin cassette (kindly provided by Prof. Paul Hudson of KTH Royal Institute of Technology of Sweden) [10, 54]. Synechocystis was transformed by electroporation $(\sim 10 \mathrm{ng}$ plasmid DNA) using GenePulser Xcell (Bio-rad, Hercules, CA) and grown photoautotrophically on agar plate adding $10 \mu \mathrm{g} / \mathrm{mL}$ kanamycin. The transformants were validated by colony PCR.

For GFP reporting assays in Synechocystis, genome sequence between sll0821 and slr0847 containing the promoter region of slr0847 was amplified through PCR and fused to GFP sequence, then the fused fragment was introduced with $K p n \mathrm{I}$ and $E c o R \mathrm{I}$ restriction site and ligated to pXT37b (digested with $K p n \mathrm{I}$ and EcoRI restriction enzymes to remove the promoter region; this vector was kindly provided by Dr. Xuefeng Lu of Qingdao Institute of Bioenergy and Bioprocess Technology of Chinese Academy of Sciences) [55]. The constructed vector was introduced into the CoaR $(+)$, CoaR $(-)$ and WT strains through electro-transformation and grown photo-autotrophically on agar plate containing $10 \mu \mathrm{g} / \mathrm{mL}$ spectinomycin with or without $10 \mu \mathrm{g} / \mathrm{mL}$ kanamycin, respectively. The transformants were validated by colony PCR and purified through passages on plates with increased antibiotics.

\section{Growth patterns and fluorescence measurement}

For measurements of growth patterns, $5 \mathrm{~mL}$ fresh cells at $\mathrm{OD}_{630}$ of 0.2 were collected by centrifugation at $3000 \times g$ and $4{ }^{\circ} \mathrm{C}$ and were then inoculated into $25 \mathrm{~mL}$ of BG11 liquid medium in a 100 -mL flask; 1-butanol treatment was carried out by adding $0.20 \%(v / v)$ 1-butanol (Merck, USA) to the medium at beginning of the cultivation, each with three replicates and repeated at least three times. Cell density was measured on a UV-1750 spectrophotometer (Shimadzu, Japan) at $\mathrm{OD}_{730}$ or on an ELx808 Absorbance Microplate Reader (BioTek, Winooski, VT, USA) at $\mathrm{OD}_{630}$. Culture samples $(1 \mathrm{~mL}$ or $200 \mu \mathrm{L}$, respectively) were taken and measured at both $\mathrm{OD}_{730}$ and $\mathrm{OD}_{630}$ every $12 \mathrm{~h}$.

For GFP reporting assays, Synechocystis strains were harvested by centrifugation $\left(4{ }^{\circ} \mathrm{C}, 7800 \times g\right)$ and re-suspended with $\mathrm{ddH}_{2} \mathrm{O}$ after $72 \mathrm{~h}$. The final OD of the re-suspensions was measured on UV-1750 
spectrophotometer and the GFP fluorescence was measured using an F-2700 fluorescence spectrophotometer (Hitachi, Japan) at EX $395 \mathrm{~nm}$ and EM $509 \mathrm{~nm}$. The data were normalized by respective OD and the fluorescence of wild type Synechocystis, each with three replicates and repeated at least three times.

\section{3'-Dephospho-CoA and CoA detection and quantitation}

The standard $\mathrm{dPCoA}$ and $\mathrm{CoA}$ were purchased from Sigma-Aldrich (St Louis, USA). The mass spectra of two substances were determined using LC/MS. The standard curve of $\mathrm{APCoA}$ and $\mathrm{CoA}$ was plotted through measuring the peak area of a series of dilutions of the respective standard, each dilution with 3 replicates using LC/MS (Additional file 1: Fig. S6). The extraction of total metabolites from Synechocystis and LC-MS analysis was conducted using the protocol described before [20]. A total of 36 samples, including WT, the $\operatorname{CoaR}(+)$ and $\operatorname{CoaR}(-)$ strains grown in normal BG11 medium or BG11 medium with $0.25 \%(v / v)$ 1-butanol were harvested at $60 \mathrm{~h}$, each with six biological replicates. The quantitation of $\mathrm{dPCoA}$ and CoA of each sample were measured through standard curve, respectively.

\section{Cell size measurement using flow cytometry}

Flow cytometric analysis was performed on a Calibur fluorescence-activated cell sorting (FACS) cytometer (Becton Dickinson) to reveal cell size differences among $\mathrm{WT}$, CoaR $(+)$ and CoaR(-) strains with the similar settings described previously [13]. Cells were harvested at $48 \mathrm{~h}$ and data analysis was conducted using the CellQuest software, version 3.1 (Becton Dickinson). The results were repeated by at least three times.

\section{Lipid profile measurement}

Total lipids from approximate 5-mg dry cells of the WT, CoaR(+), and CoaR(-) strains (each with 3 biological replicates) were extracted using $2 \mathrm{~mL}$ chloroform/ methanol $(v / v, 2 / 1)$ ultrasonic treatment for $10 \mathrm{~min}$ and centrifugation at $4000 \mathrm{rpm}$ for $5 \mathrm{~min}$. The collected supernatants were dried under nitrogen flow and then at $60^{\circ} \mathrm{C}$ until the weight of samples remained constant. The samples were weighed and moved to $10-\mathrm{mL}$ flasks. Then, $5 \mathrm{~mL} 2.0 \% \mathrm{H}_{2} \mathrm{SO}_{4}$-methanol ( $v / v, \mathrm{H}_{2} \mathrm{SO}_{4} /$ methanol) was added, and the flask was stirred at $70{ }^{\circ} \mathrm{C}$ for $1 \mathrm{~h}$. Then $2 \mathrm{~mL}$ of hexane and $0.75 \mathrm{~mL}$ of distilled water were added to the flask and mixed and the upper hexane layer contained the fatty acid methyl esters (FAMEs). The hexane layer was transferred to a new vial and mixed with the internal standard C17-ME for analysis by gas chromatography (GC). FAME analyses were carried out by an Agilent $6890 \mathrm{GC}$ instrument and FAME yield was calculated using the equation described by Liu et al. [32].

\section{Quantitative proteomics and data analysis}

Samples composed of the WT and the mutant CoaR $(+)$ were harvested after cultivation for $48 \mathrm{~h}$ under $0.20 \%$ $(v / v)$ 1-butanol stress, each with two replicates for quantitative isobaric tags for relative and absolute quantification (iTRAQ) liquid chromatography-tandem mass spectrometry (LC-MS/MS) proteomic analysis. Protein preparation and digestion, iTRAQ labeling, LC-MS/ MS proteomic analysis, and proteomic data analysis were the same as before [36]. Briefly, cell samples for proteomics analysis were collected by centrifugation at $8000 \times g$ for $10 \mathrm{~min}$ at $4{ }^{\circ} \mathrm{C}$ and immediately frozen in liquid nitrogen. After proteins preparation, the iTRAQ labeling of peptide samples was performed using iTRAQ Reagent 8-plex Kit (Applied Biosystems, Foster City, CA) according to the manufacturer's protocol. Then the mass spectroscopy analysis was performed using an AB SCIEX TripleTOF ${ }^{\mathrm{TM}} 5600$ mass spectrometer (AB SCIEX, Framingham, MA, USA), coupled with online micro flow HPLC system (Shimadzu, JAPAN). The MS data were processed using Proteome Discoverer software (Version 1.2.0.208) (Thermo Scientific) with default parameters to generating peak list. Genome sequence and annotation information of Synechocystis were downloaded from NCBI and the Comprehensive Microbial Resource (CMR) of TIGR (http://www.tigr.org/CMR) [56]. Proteins with 1.2-fold change between the CoaR $(+)$ strain and control WT samples and $p$-value of statistical evaluation $<0.05$ were determined as differentially expressed proteins.

\section{Additional files}

Additional file 1: Fig. S1. Mutant constructions using different vectors. A) Overexpression of sRNAs using pJA2. B) Suppression of sRNAs using pJA2. C) Gene overexpression using pXT37b. Fig. S2 A) Growth phenotypes of WT, Ncl1460(+), and Ncl1460(-) under BG11 media with $4.6 \mu \mathrm{M}$ $\mathrm{CdSO}_{4}$; B) Growth phenotypes of WT, Ncl1460(+), and Ncl1460(-) under BG11 media under pH6.1; C) Growth phenotypes of WT, Ncl1460(+), and $\mathrm{Ncl} 1460(-)$ under BG11 media with $4 \%(\mathrm{~W} / \mathrm{V}) \mathrm{NaCl}$. Fig. S3 A) Northern blotting detection of NCl1460. B) 3'RACE results of NCl1460. C) RT-PCR results of Ncl1460 in WT, Ncl1460(+), and Ncl1460(-). Fig. S4 Predicted functional regions of Ncl1460 using CopraRNA. Fig. S5 Predicted interaction regions of $\mathrm{Ncl} 1460$ on its target genes using CopraRNA. Fig. S6 Standard curves for measurements of (OA A) and dPCOA B). Fig. S7 Description of the proteomics data. A) Basic information statistics including the total spectrum, unique spectrum, peptides, unique peptides, and identified proteins. B) Peptide length distribution. C) Distribution of protein's sequences coverage. The different colors represent different coverages, and the number in each color represents the identified proteins.

Additional file 2: Table S1. Mapping results and the expression profiles of all identified sRNAs. C represents Synechocystis cultured in normal BG11 while $B$ represents medium with butanol. The RPKM value was reads per kilobase per million mapped reads. The fold change of each ncRNAs was shown as B/C. Table S2 SRNAs with regulated expression at $24 \mathrm{~h}$, $48 \mathrm{~h}$, and $72 \mathrm{~h}$. Table S3 Primers used in this study. Table $\mathbf{S 4}$ Constructed sRNA mutants in this study. Table $\mathbf{S 5}$ Regulated proteins by CoaR. Table S6 plasmids and strains constructed in this study. 


\section{Abbreviations}

dPCoA: 3'-dephospho-CoA; LC/MS: liquid chromatography-mass spectrometry; qRT-PCR: quantitative real time polymerase chain reaction; RT-PCR: reverse transcriptional polymerase chain reaction; 3'RACE: $3^{\prime}$ rapid amplification of CDNA end; RPKM: reads per kilobase per million mapped reads; sRNA: small RNA; sRNome: small RNA transcriptome.

\section{Authors' contributions}

TS performed the experiments and wrote the manuscript; TS, GP, JW, LC and WZ analyzed the data; LC and WZ designed and revised this paper. All authors read and approved the final manuscript.

\begin{abstract}
Author details
${ }^{1}$ Laboratory of Synthetic Microbiology, School of Chemical Engineering and Technology, Tianjin University, Tianjin 300072, People's Republic of China ${ }^{2}$ Key Laboratory of Systems Bioengineering (Ministry of Education), Tianjin University, Tianjin 300072, People's Republic of China. ${ }^{3}$ SynBio Research Platform, Collaborative Innovation Center of Chemical Science and Engineering, Tianjin 300072, People's Republic of China. ${ }^{4}$ Shenzhen Engineering Lab for Marine Algal Biotechnology, College of Life Science, Shenzhen University, Shenzhen 518060, People's Republic of China. ${ }^{5}$ Center for Biosafety Research
\end{abstract} and Strategy, Tianjin University, Tianjin, People's Republic of China.

\section{Acknowledgements}

We sincerely thank Prof. Song Xue and Dr. Jiao Liu from Dalian Institute of Chemical Physics, Chinese Academy of Sciences for the help with lipid profile analysis. We also sincerely thank Zixi Chen and Xiaoqing Zhang for the flow cytometry analysis and CoA quantification, respectively.

\section{Competing interests}

The authors declare that they have no competing interests.

\section{Availability of supporting data}

All data generated or analyzed during this study are included in this published article and its additional files.

\section{Additional information}

The raw small RNA sequence data of Synechocystis are deposited in the SRA database of NCBI with accession numbers SRP073279.

\section{Consent for publication}

All authors agree to publication.

\section{Funding}

This research was supported by grants from the National Science Foundation of China (No. 31470217 and No. 21621004), the National Basic Research Program of China ("973" program, project No. 2014CB745101), the National High-Tech R\&D Program ("863" program, project No. 2012AA02A707), and the Tianjin Municipal Science and Technology Commission (No. 15JCZDJC32500).

Received: 17 November 2016 Accepted: 9 February 2017 Published online: 20 February 2017

\section{References}

1. Storz G, Vogel J, Wassarman KM. Regulation by small RNAs in bacteria: expanding frontiers. Mol Cell. 2011;43:880-91.

2. Gao X, Sun T, Pei G, Chen L, Zhang W. Cyanobacterial chassis engineering for enhancing production of biofuels and chemicals. Appl Microbiol Biotechnol. 2016;100:3401-13.

3. Mitschke J, Georg J, Scholz I, Sharma CM, Dienst D, Bantscheff J, Voss B, Steglich C, Wilde A, Vogel J, Hess WR. An experimentally anchored map of transcriptional start sites in the model cyanobacterium Synechocystis sp. PCC6803. Proc Natl Acad Sci. 2011;108:2124-9.

4. Kopf M, Hess WR. Regulatory RNAs in photosynthetic cyanobacteria. FEMS Microbiol Rev. 2015;39:301-15.

5. Klahn S, Schaal C, Georg J, Baumgartner D, Knippen G, Hagemann M, Muro-Pastor AM, Hess WR. The sRNA NsiR4 is involved in nitrogen assimilation control in cyanobacteria by targeting glutamine synthetase inactivating factor IF7. Proc Natl Acad Sci USA. 2015;112(45):E6243-52.
6. Lan El, Ro SY, Liao JC. Oxygen-tolerant coenzyme A-acylating aldehyde dehydrogenase facilitates efficient photosynthetic n-butanol biosynthesis in cyanobacteria. Energy Environ Sci. 2013;6:2672.

7. Anfelt J, Kaczmarzyk D, Shabestary K, Renberg B, Rockberg J, Nielsen J, Uhlen M, Hudson EP. Genetic and nutrient modulation of acetylCoA levels in Synechocystis for n-butanol production. Microb Cell Fact. 2015;14:167.

8. Luo H, Ge L, Zhang J, Ding J, Chen R, Shi Z. Enhancing acetone biosynthesis and acetone-butanol-ethanol fermentation performance by coculturing Clostridium acetobutylicum/Saccharomyces cerevisiae integrated with exogenous acetate addition. Bioresour Technol. 2016;200:111-20.

9. Shen CR, Lan El, Dekishima Y, Baez A, Cho KM, Liao JC. Driving forces enable high-titer anaerobic 1-butanol synthesis in Escherichia coli. Appl Environ Microbiol. 2011;77:2905-15.

10. Kaczmarzyk D, Anfelt J, Sarnegrim A, Hudson EP. Overexpression of sigma factor SigB improves temperature and butanol tolerance of Synechocystis sp. PCC6803. J Biotechnol. 2014;182-183:54-60.

11. Chen L, Wu L, Wang J, Zhang W. Butanol tolerance regulated by a twocomponent response regulator SIr1037 in photosynthetic Synechocystis sp. PCC 6803proteomic. Biotechnol Biofuels. 1037;2014:7.

12. Niu X, Zhu Y, Pei G, Wu L, Chen L, Zhang W. Elucidating butanol tolerance mediated by a response regulator SII0039 in Synechocystis sp. PCC 6803 using a metabolomic approach. Appl Microbiol Biotechnol. 2015;99:1845-57.

13. Song Z, Chen L, Wang J, Lu Y, Jiang W, Zhang W. A transcriptional regula-

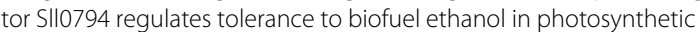
Synechocystis sp. PCC 6803. Mol Cell Proteomics. 2014;13(12):3519-32.

14. Zhu Y, Pei G, Niu X, Shi M, Zhang M, Chen L, Zhang W. Metabolomic analysis reveals functional overlapping of three signal transduction proteins in regulating ethanol tolerance in cyanobacterium Synechocystis sp. PCC 6803. Mol Biosyst. 2015;11(3):770-82

15. Gaida SM, Al-Hinai MA, Indurthi DC, Nicolaou SA, Papoutsakis ET. Synthetic tolerance: three noncoding small RNAs, DsrA, ArcZ and RprA, acting supra-additively against acid stress. Nucleic Acids Res. 2013;41:8726-37.

16. Dienst D, Georg J, Abts T, Jakorew L, Kuchmina E, Boerner T, Wilde A, Duehring $U$, Enke $H$, Hess WR. Transcriptomic response to prolonged ethanol production in the cyanobacterium Synechocystis sp. PCC6803. Biotechnol Biofuels. 2014;7:21.

17. Zhu H, Ren X, Wang J, Song Z, Shi M, Qiao J, Tian X, Liu J, Chen L, Zhang W. Integrated OMICS guided engineering of biofuel butanol-tolerance in photosynthetic Synechocystis sp. PCC 6803. Biotechnol Biofuels. 2013;6:106.

18. Sakurai I, Stazic D, Eisenhut M, Vuorio E, Steglich C, Hess WR, Aro EM. Positive regulation of psbA gene expression by cis-encoded antisense RNAs in Synechocystis sp. PCC 6803. Plant Physiol. 2012;160:1000-10.

19. Kopf M, Klahn S, Scholz I, Matthiessen JK, Hess WR, Voss B: Comparative Analysis of the Primary Transcriptome of Synechocystis sp. PCC 6803. DNA Res 2014.

20. Wang Y, Shi M, Niu X, Zhang X, Gao L, Chen L, Wang J, Zhang W. Metabolomic basis of laboratory evolution of butanol tolerance in photosynthetic Synechocystis sp. PCC 6803. Microb Cell Fact. 2014;13:151.

21. Wright PR, Georg J, Mann M, Sorescu DA, Richter AS, Lott S, Kleinkauf R, Hess WR, Backofen R. CopraRNA and IntaRNA: predicting small RNA targets, networks and interaction domains. Nucleic Acids Res. 2014;42:W119-23.

22. Georg J, Dienst D, Schurgers N, Wallner T, Kopp D, Stazic D, Kuchmina E, Klahn S, Lokstein H, Hess WR, Wilde A. The small regulatory RNA SyR1/ PsrR1 controls photosynthetic functions in cyanobacteria. Plant Cell. 2014;26(9):3661-79.

23. Knoop H, Zilliges Y, Lockau W, Steuer R. The metabolic network of Synechocystis sp. PCC 6803: systemic properties of autotrophic growth. Plant Physiol. 2010;154:410-22.

24. Price MN, Huang KH, Alm EJ, Arkin AP. A novel method for accurate operon predictions in all sequenced prokaryotes. Nucleic Acids Res. 2005;33:880-92.

25. Marbouty M, Saguez C, Cassier-Chauvat C, Chauvat F. ZipN, an FtsA-like orchestrator of divisome assembly in the model cyanobacterium Synechocystis PCC6803. Mol Microbiol. 2009;74:409-20.

26. Yan BX, Ma JX. Promoter-associated RNAs and promoter-targeted RNAs. Cell Mol Life Sci. 2012;69:2833-42. 
27. Morris KV, Chan SW, Jacobsen SE, Looney DJ. Small interfering RNA-induced transcriptional gene silencing in human cells. Science. 2004;305:1289-92.

28. Begley TP, Kinsland C, Strauss E. The biosynthesis of coenzyme A in bacteria. Vitam Horm. 2001;61:157-71.

29. Ling $H$, Chen B, Kang A, Lee JM, Chang MW. Transcriptome response to alkane biofuels in Saccharomyces cerevisiae: identification of efflux pumps involved in alkane tolerance. Biotechnol Biofuels. 2013;6:95.

30. Oefner C, Schulz H, D'Arcy A, Dale GE. Mapping the active site of Escherichia coli malonyl-CoA-acyl carrier protein transacylase (FabD) by protein crystallography. Acta Crystallogr D Biol Crystallogr. 2006;62:613-8.

31. Burdette DS, Jung SH, Shen GJ, Hollingsworth RI, Zeikus JG. Physiological function of alcohol dehydrogenases and long-chain (C(30)) fatty acids in alcohol tolerance of Thermoanaerobacter ethanolicus. Appl Environ Microbiol. 2002;68:1914-8.

32. Liu J, Liu Y, Wang H, Xue S. Direct transesterification of fresh microalgal cells. Bioresour Technol. 2015;176:284-7.

33. Lei A, Chen H, Shen G, Hu Z, Chen L, Wang J. Expression of fatty acid synthesis genes and fatty acid accumulation in haematococcus pluvialis under different stressors. Biotechnol Biofuels. 2012;5:18.

34. Rowland JG, Simon WJ, Nishiyama Y, Slabas AR. Differential proteomic analysis using ITRAQ reveals changes in thylakoids associated with Photosystem II-acquired thermotolerance in Synechocystis sp. PCC 6803. Proteomics. 2010;10:1917-29.

35. Howitt CA, Udall PK, Vermaas WF. Type 2 NADH dehydrogenases in the cyanobacterium Synechocystis sp. strain PCC 6803 are involved in regulation rather than respiration. J Bacteriol. 1999;181:3994-4003.

36. Tian X, Chen L, Wang J, Qiao J, Zhang W. Quantitative proteomics reveals dynamic responses of Synechocystis sp. PCC 6803 to next-generation biofuel butanol. J Proteomics. 2013;78:326-45.

37. Wang J, Chen L, Huang S, Liu J, Ren X, Tian X, Qiao J, Zhang W. RNA-seq based identification and mutant validation of gene targets related to ethanol resistance in cyanobacterial Synechocystis sp. PCC 6803. Biotechnol Biofuels. 2012;5:89.

38. Foo JL, Jensen HM, Dahl RH, George K, Keasling JD, Lee TS, Leong S, Mukhopadhyay A. Improving microbial biogasoline production in Escherichia coli using tolerance engineering. MBio. 2014;5:e01932.

39. Los DA, Zorina A, Sinetova M, Kryazhov S, Mironov K, Zinchenko W. Stress sensors and signal transducers in cyanobacteria. Sensors. 2010;10:2386-415.

40. Srinivasan B, Baratashvili M, van der Zwaag M, Kanon B, Colombelli C, Lambrechts RA, Schaap O, Nollen EA, Podgorsek A, Kosec G, et al. Extracellular 4'-phosphopantetheine is a source for intracellular coenzyme A synthesis. Nat Chem Biol. 2015;11:784-92.

41. Rubio S, Whitehead L, Larson TR, Graham IA, Rodriguez PL. The coenzyme a biosynthetic enzyme phosphopantetheine adenylyltransferase plays a crucial role in plant growth, salt/osmotic stress resistance, and seed lipid storage. Plant Physiol. 2008;148:546-56.

42. Leonardi R, Zhang YM, Rock CO, Jackowski S. Coenzyme A: back in action Prog Lipid Res. 2005;44:125-53.
43. Rock CO, Calder RB, Karim MA, Jackowski S. Pantothenate kinase regulation of the intracellular concentration of coenzyme A. J Biol Chem. 2000;275:1377-83.

44. Geerlof A, Lewendon A, Shaw WV. Purification and characterization of phosphopantetheine adenylyltransferase from Escherichia coli. J Biol Chem. 1999;274:27105-11.

45. Chatterjee R, Mondal A, Basu A, Datta S. Transition of phosphopantetheine adenylyltransferase from catalytic to allosteric state is characterized by ternary complex formation in Pseudomonas aeruginosa. Biochim Biophys Acta. 2016;1864:773-86.

46. Venkataramanan KP, Jones SW, McCormick KP, Kunjeti SG, Ralston MT, Meyers BC, Papoutsakis ET. The Clostridium small RNome that responds to stress: the paradigm and importance of toxic metabolite stress in $C$. acetobutylicum. BMC Genom. 2013;14:849.

47. Cho SH, Lei R, Henninger TD, Contreras LM. Discovery of ethanolresponsive small RNAs in Zymomonas mobilis. Appl Environ Microbiol. 2014;80:4189-98.

48. Aguilar C, Escalante A, Flores N, de Anda R, Riveros-McKay F, Gosset G, Morett E, Bolivar F. Genetic changes during a laboratory adaptive evolution process that allowed fast growth in glucose to an Escherichia coli strain lacking the major glucose transport system. BMC Genom. 2012;13:385.

49. Ter Beek A, Keijser BJ, Boorsma A, Zakrzewska A, Orij R, Smits GJ, Brul S. Transcriptome analysis of sorbic acid-stressed Bacillus subtilis reveals a nutrient limitation response and indicates plasma membrane remodeling. J Bacteriol. 2008;190:1751-61.

50. Soini J, Falschlehner C, Mayer C, Bohm D, Weinel S, Panula J, Vasala A, Neubauer P. Transient increase of ATP as a response to temperature upshift in Escherichia coli. Microb Cell Fact. 2005:4:9.

51. Fleury B, Kelley WL, Lew D, Gotz F, Proctor RA, Vaudaux P. Transcriptomic and metabolic responses of Staphylococcus aureus exposed to supraphysiological temperatures. BMC Microbiol. 2009;9:76.

52. Livak KJ, Schmittgen TD. Analysis of relative gene expression data using real-time quantitative PCR and the 2(-Delta Delta C(T)) Method. Methods. 2001;25:402-8

53. Kim SW, Li Z, Moore PS, Monaghan AP, Chang Y, Nichols M, John B. A sensitive non-radioactive northern blot method to detect small RNAs. Nucleic Acids Res. 2010:38:e98.

54. Huang HH, Camsund D, Lindblad P, Heidorn T. Design and characterization of molecular tools for a Synthetic Biology approach towards developing cyanobacterial biotechnology. Nucleic Acids Res. 2010;38:2577-93.

55. Tan X, Yao L, Gao Q, Wang W, Oi F, Lu X. Photosynthesis driven conversion of carbon dioxide to fatty alcohols and hydrocarbons in cyanobacteria. Metab Eng. 2011;13:169-76.

56. Kaneko T, Nakamura Y, Sasamoto S, Watanabe A, Kohara M, Matsumoto M, Shimpo S, Yamada M, Tabata S. Structural analysis of four large plasmids harboring in a unicellular cyanobacterium, Synechocystis sp. PCC 6803. DNA Res. 2003;10:221-8.

\section{Submit your next manuscript to BioMed Central and we will help you at every step:}

- We accept pre-submission inquiries

- Our selector tool helps you to find the most relevant journal

- We provide round the clock customer support

- Convenient online submission

- Thorough peer review

- Inclusion in PubMed and all major indexing services

- Maximum visibility for your research

Submit your manuscript at www.biomedcentral com/submit 